\title{
Freqüência de Giardia lamblia em cães atendidos em clínicas veterinárias de Porto Alegre, RS, Brasil
}

\author{
Frequency of Giardia lamblia in dogs attended by veterinary clinics in \\ Porto Alegre city, RS, Brazil
}

\author{
Adriane Bartmann ${ }^{1}$ Flávio Antônio Pacheco de Araújo
}

\section{RESUMO}

A pesquisa de cistos de Giardia lamblia em fezes de cães oriundos de clínicas veterinárias de Porto Alegre, RS, Brasil, foi realizada pelo método de FAUST et al. (1939). Das 526 amostras analisadas, 38\% (198) foram positivas para cistos de G. lamblia, sendo $22 \%$ (116) constituídos por animais menores de 11 meses de idade e 16\% (82) constituídos por animais com 12 meses ou mais de idade. Houve diferença significativa $(p<0,05)$ entre os grupos pelo teste do $\chi^{2}$. Dos animais pesquisados, $54 \%$ (285) eram machos, e, destes, $20 \%$ (104) apresentaram cistos em suas fezes. Dos $46 \%$ (241) de fêmeas analisadas, 18\% (95) tiveram suas amostras positivas para cistos de G. lamblia, não havendo diferença significativa entre os resultados encontrados pelo teste do $\chi^{2}$. Os animais jovens (menores de 11 meses de idade) apresentaram uma taxa de positividade duas vezes maior que os animais com idade superior a 12 meses, e a variável sexo não apresentou associação com a positividade.

Palavras-chave: Giardia lamblia, cães, epidemiologia.

\section{ABSTRACT}

The method of Faust et al. (1939) was utilized for detection of Giardia lamblia cysts in feces from dogs in Porto Alegre city, Rio Grande Sul State, Brazil. From the 526 analyzed samples $38 \%$ (198) showed positive results for the presence of $\mathbf{G}$. lamblia cyst, so $22 \%$ (116) belonged to group one, formed by animals that were less than eleven months old; and $16 \%$ (82) belonged to group two, formed by animals that were twelve months or older. There was a significant difference $(p<0,05)$ among the two groups checked by the $\div^{2}$ test. From the animals researched, $54 \%$ (285) were male dogs and from these $20 \%$ (104) presented cysts in their feces. From the other $46 \%$ (241), which were female dogs, $18 \%$ (95) presented positive results for the cysts of $\mathbf{G}$. lamblia in their sample. The $\div^{2}$ test applied to these data showed that there was not any significant difference between the results found in female or male dogs of all ages. Based on these results, we can come to the conclusion that $38 \%$ of the analyzed dogs showed positive results for the infection of G. lamblia and that the younger animals those aged less than eleven months presented a rate of risk to get infected which is two times bigger than the ones for the animals with 12 months or older, as well as, the fact that the sex of the animals did not present any association to the positive results of infection.

Key words: Giardia lamblia, dogs, epidemiology.

\section{INTRODUÇÃO}

O gênero Giardia foi descrito por Kunstler em 1882, ao observar um flagelado presente no intestino de girinos de anfíbios anuros. Este gênero é constituído por protozoários cosmopolitas que ocorrem principalmente em regiões tropicais, mas pode ser encontrado em temperaturas variadas. Existem evidências de que Giardia lamblia não apresenta especificidade quanto ao hospedeiro e pode parasitar seres humanos, assim como uma variedade de outros animais, sendo considerada uma importante zoonose (SOGAYAR \& GUIMARÃES, 2000).

No sul da Alemanha as infecções por Giardia ocorrem com maior freqüência em filhotes de cães e em canis ou em estabelecimentos de criação intensa, do que em animais mais velhos, cães mantidos individualmente como animais de estimação ou cães de canis de pequeno porte (BARUTZKI et al., 2000).

\footnotetext{
${ }^{1}$ Médico Veterinário, Mestre, Professor da Faculdade de Medicina Veterinária da Universidade do Oeste de Santa Catarina (UNOESC), Xanxerê, SC, Brasil. E-mail: badriane@bol.com.br Endereço: Av. Carazinho, 501, apt. 413, Bairro Petrópolis, 90460-190, Porto Alegre, RS.

${ }^{2}$ Médico Veterinário, Doutor, Professor, Faculdade de Medicina Veterinária, Setor de Protozoologia Veterinária. Depto Parasitologia Veterinária, Universidade Federal do Rio Grande do Sul (UFRGS), Av. Bento Gonçalves, 9090, CP 15094, 91540-000, Porto Alegre, RS. E-mail: faraujo@vortex.ufrgs.br.
} 
Este estudo se justifica por ser a giardíase uma enfermidade com características zoonóticas. Análises moleculares têm demonstrado o mesmo genótipo de Giardia spp presente em humanos e em outras espécies de mamíferos (THOMPSON et al., 2000). CONNAUGHTON (1989), THOMPSON et al. (1990), ZAJAC (1992), GIANGASPERO et al. (2000), SOGAYAR \& GUIMARÃES (2000) também descreveram o potencial de transmissão de Giardia aos humanos pelos animais, principalmente aos imunodeficientes, considerando cães e gatos parasitados como origem de infecções humanas. O presente trabalho objetivou analisar a freqüência de Giardia em cães atendidos em clínicas de Porto Alegre.

\section{MATERIAL E MÉTODOS}

Amostragem - Foram coletadas amostras fecais de cães atendidos em 38 diferentes estabelecimentos veterinários, incluindo clínicas ou hospitais veterinários e atendimentos domiciliares realizados pelos médicos veterinários envolvidos, na cidade de Porto Alegre, RS, Brasil, nos anos de 2001 e 2002.

Animais - Participaram da pesquisa 526 cães, independentes de sintomatologia para giardíase, mas que apresentaram suspeita de parasitismo, seja pelo histórico ou pelo exame clínico. Os cães pertenciam a diferentes raças, ambos os sexos, os quais foram categorizados de acordo com o sexo e a idade obtidos por informações do proprietário, em dois grupos: Grupo 1, constituído por animais com idade menor ou igual a 11 meses. Grupo 2, constituído por animais com idade igual ou maior que 12 meses. Os animais foram separados ainda quanto ao sexo.

Material coletado - Amostra fecal de cada animal foi coletada no momento da sua defecação, sendo armazenada sob refrigeração, em potes plásticos limpos apropriados para este fim e devidamente identificados. As amostras foram encaminhadas ao Laboratório de Protozoologia da UFRGS e ao
Laboratório de Análises Clínicas Veterinárias PETLAB, onde foram analisadas até seis horas após o recebimento.

Técnica utilizada - Para diagnosticar a presença de cistos de G. lamblia nas fezes, foi utilizado o método de Faust et al. (1939), segundo HOFFMANN (1987). O diagnóstico foi baseado na presença de cistos e/ou trofozoítos de G. lamblia nas fezes.

Análise estatística - A avaliação estatística foi definida pela utilização do teste de Qui-quadrado $\left(\chi^{2}\right)$ com a correção de Yates, a $O d d s$ Ratio e a análise de regressão logística foram realizadas considerando-se como variável resposta à presença ou ausência de cistos de Giardia spp nas amostras de fezes caninas. Foram consideradas como significativas as diferenças cuja probabilidade de erro $\propto$ fosse menor que $5 \%$ $(\mathrm{p}<0,05)$ (THRUSFIELD, 1986).

\section{RESULTADOS}

Após a análise das amostras fecais de todos os cães, constatou-se que 198 (37,64\%) animais apresentaram cistos de G. lamblia em suas fezes. A estimativa de ponto do valor populacional da freqüência foi de $37,64 \%$ e para um intervalo de confiança de $95 \%$, usando-se aproximação normal variou entre $33,50 \%$ a $41,78 \%$. Desta forma, a positividade para $\boldsymbol{G}$. lamblia nas clínicas veterinárias da cidade de Porto Alegre, RS, variou entre 33\% e $42 \%$. Por outro lado, o número de amostras negativas chegou a $328(62,17 \%)$ do total analisado, conforme pode-se observar na tabela 1 .

O teste do $\chi^{2}$ aplicado aos dados da Tabela 1 , revelou haver diferença significativa entre os resultados dos grupos de animais jovens e adultos $(\mathrm{p}=0,0003)$.

A Odds Ratio calculada a partir dos dados encontrados foi igual a 1,972, o que significa dizer que os animais pertencentes ao grupo 1 - animais menores ou iguais a 11 meses de idade, apresentaram uma taxa de positividade aproximadamente duas vezes maior

Tabela 1 - Freqüências absolutas de Giardia lamblia conforme a faixa etária e o sexo de cães atendidos em clínicas veterinárias de Porto Alegre, nos anos de 2001 e 2002.

\begin{tabular}{|c|c|c|c|c|c|c|}
\hline \multirow{2}{*}{$\begin{array}{l}\text { Idade } \\
\text { (meses) }\end{array}$} & \multicolumn{2}{|c|}{ Machos } & \multicolumn{2}{|c|}{ Fêmeas } & \multicolumn{2}{|c|}{ Total } \\
\hline & $\mathrm{n}$ & positivos & $\mathrm{n}$ & positivos & $\mathrm{n}$ & positivos \\
\hline$\leq 11$ & 133 & 58 & 120 & 58 & 253 & 116 \\
\hline$\geq 12$ & 151 & 45 & 122 & 37 & 273 & 82 \\
\hline Total & 284 & 103 & 242 & 95 & 526 & 198 \\
\hline
\end{tabular}


que os animais do grupo 2 - animais maiores ou iguais a 12 meses de idade.

Em função do sexo dos animais, dos 284 machos analisados, 103 (36,27\%) apresentaram amostras com resultado positivo para presença do protozoário G. lamblia. E das 242 fêmeas analisadas, $95(39,26 \%)$ foram positivas para o mesmo parasito (Tabela 1). Não houve diferença significativa entre os resultados, quanto a machos e fêmea, dos dois grupos etários estudados $(\mathrm{p}=0,5487)$. A Odds Ratio calculada a partir dos dados encontrados foi igual a 0,8830 , o que indica possivelmente, que a taxa de positividade é semelhante em ambos os sexos (Tabela 2).

\section{DISCUSSÃO E CONCLUSÃO}

Por meio do exame de fezes, pode-se encontrar cistos de Giardia spp na maioria dos animais infectados com o protozoário, ao passo que os trofozoítos são raramente visualizados, porque se tornam inviáveis em poucos minutos após a eliminação do hospedeiro. As amostras de fezes podem ser processadas, entre outras técnicas, pelo método de flutuação em sulfato de zinco (ADAM, 1991). Em geral, é a melhor técnica para identificação, e a que apresenta maior positividade para cistos de Giardia spp, permitindo ser utilizada na rotina laboratorial (ZAJAC, 1992; LORENZ \& FERGUSON, 1996; OLIVEIRASEQUEIRA et al., 2002). De acordo com ADAM (1991), HERESI \& CLEARY (1997) e RODRIGUES et al. (2001), a repetição de amostras é importante, uma vez que, segundo esses autores, uma das limitações da técnica de flutuação em sulfato de zinco é a sensibilidade que varia de $50 \%$ a $70 \%$ para o exame de apenas uma amostra. Essa sensibilidade aumenta para $95 \%$ se caso duas ou três amostras de fezes forem analisadas, com coletas em dias alternados.

$\mathrm{Na}$ rotina da clínica veterinária, alguns fatores, como disponibilidade de tempo do proprietário e até o custo, podem inviabilizar a realização de duas ou três amostras como é o indicado.
Deste ponto de vista, pode-se pensar que os presentes resultados poderiam ser maiores se fosse utilizada a metodologia proposta. Neste trabalho, evidenciou-se que, na análise de apenas uma amostra, há probabilidade de positividade para G. lamblia de até $42 \%$.

BURROWS \& LILLIS (1967), HAHN et al. (1988), SCHANTZ (1999) e GIANGASPERO et al. (2000) relataram não existir diferença significativa entre machos e fêmeas para infecções com Giardia spp, resultado concordante com os do presente trabalho. Estes autores, também verificaram que as maiores freqüências de positividade para G. lamblia ocorreram em cães menores de 11 meses de idade quando comparados com cães adultos, também de acordo com o encontrado no presente trabalho.

Com base nos dados obtidos através da análise laboratorial pelo método de Faust et al. (1939), em fezes caninas enviadas para exame parasitológico por clínicas veterinárias da cidade de Porto Alegre, $\mathrm{RS}$, pode-se concluir que a giardíase ocorre com freqüência em cães atendidos na rotina de clínicas e laboratórios veterinários da cidade de Porto Alegre. A freqüência estimada de $\boldsymbol{G}$. lamblia em exames parasitológicos em fezes de animais suspeitos de parasitismo gastrintestinal, requisitados por médicos veterinários, situa-se entre $33,50 \%$ e $41,78 \%$. Animais com idade igual a 11 meses apresentam uma taxa de positividade maior que os animais com idade igual a 12 meses. O método de FAUST et al. (1939) deve ser mantido entre as técnicas utilizadas na rotina laboratorial para o diagnóstico específico de Giardia spp, mesmo com a utilização de apenas uma amostra fecal.

\section{AGRADECIMENTOS}

Expressamos nossos agradecimentos às pessoas e às instituições que colaboraram no desenvolvimento deste trabalho, a Reginaldo José Diaz Rodrigues, a Márcia Regina Oliveira Cordeiro e a professora Dra. Vera Wald.

Tabela 2 - Análise de regressão logística para a presença de Giardia lamblia, conforme a faixa etária e o sexo de cães atendidos em clínicas veterinárias.

\begin{tabular}{|c|c|c|c|c|c|}
\hline \multirow{2}{*}{ Preditores } & \multirow{2}{*}{$\beta$} & \multirow{2}{*}{$\mathrm{p}$} & \multirow{2}{*}{ Odds Ratio (O R) } & \multicolumn{2}{|c|}{$\mathrm{IC}^{*} 95 \%$ do O R } \\
\hline & & & & inferior & superior \\
\hline Constante & 0,22 & 0,152 & & & \\
\hline Faixa etária & 0,68 & 0,000 & 1,97 & 1,38 & 2,81 \\
\hline Sexo & $-0,11$ & 0,542 & 0,89 & 0,62 & 1,28 \\
\hline
\end{tabular}

* I. C. - Intervalo de Confiança. 


\section{REFERÊNCIAS BIBLIOGRÁFICAS}

ADAM, R.D. The biology of Giardia spp. Microbiological Review, v.55, n.4, p.706-732, 1991.

BARUTZKI, D.; SCHIMMEL, A.; SCHAPER, R. Eficácia de pamoato de pirantel, febantel e praziquantel contra Giardia em cães naturalmente contaminados. [Brasil]: Bayer, [2000]. p.5-7. (Giardia: Informativo Técnico).

BURROWS, R.B.; LILLIS, W.G. Intestinal protozoan infections in dogs. Journal American Veterinary Medical Association, v.150, n.8, p.880-883, 1967.

CONNAUGHTON, D. Giardiasis - zoonosis or not? Journal American Veterinary Medical Association, v.194, n.4, p.447-451, 1989.

GIANGASPERO, A.; TRALDI, G.; BIANCIARDI, P. Avaliação da eficiência do tratamento com pamoato de pirantel, febantel e praziquantel contra Giardia em cães adultos naturalmente infectados. [Brasil.] : Bayer, [2000]. p.9-11. (Giardia: informativo técnico).

HAHN, N.E. et al. Prevalence of Giardia in the feces of pups. Journal American Veterinary Medical Association, v.192, n.10, p.1428-1429, 1988.

HERESI, G.; ClEARY, T.G. Giardia. Pediatrics in Review, v.18, n.7, p.243-247, 1997.

HOFFMANN, R.P. Diagnóstico de parasitismo veterinário. Porto Alegre : Sulina, 1987. 156p.

LORENZ, M.D.; FERGUSON, D.C. Doenças do sistema digestivo. In: LORENZ, M.D.; CORNELIUS, L.M.;
FERGUSON, D.C. Terapêutica clínica em pequenos animais. Rio de Janeiro : Interlivros, 1996. Cap.8, p.175199.

OLIVEIRA - SEQUEIRA, T.C.G. et al. Prevalence of intestinal parasites in doges from São Paulo State, Brazil. Veterinary Parasitology, v.103, p.19-27, 2002.

RODRIGUES, L.C.S. et al. Importância da repetição de exames coproscópicos na avaliação da prevalência de parasitoses intestinais. Jornal Brasileiro de Patologia, Rio de Janeiro, v.37, n.4, 2001. (Suplemento XV Congresso Latino Americano de Parasitologia).

SCHANTZ, P.M. Intestinal parasites of dogs in Western Austrália: progress in control and new concerns. Veterinary Journal, v.157, n.3, p.222224, 1999 .

SOGAYAR, M.I.T.L.; GUIMARÃES, S. Giardia lamblia. In: NEVES, D.P., et al. Parasitologia humana. 10.ed. São Paulo : Atheneu, 2000. p.107-113.

THOMPSON, R.C.A. et al. The zoonotic transmission of Giardia species. Veterinary Record, v.19, p.513-514, 1990.

THOMPSON, R.C.A.; HOPKINS, R.M.; HOMAN, W.L. Nomenclature and genetic groupings of Giardia infecting mammals. Parasitology Today, v.16, n.5, p.210-213, 2000.

THRUSFIELD, M. Veterinary epidemiology. Great Britain : Butterworth, 1986. 280p.

ZAJAC, A.M. Giardiasis. Compendium on Continuing Education for de Practicing veterinarian - Small Animal, v.14, n.5, p.604-611, 1992. 